\title{
Extrasolar planets and brown dwarfs around A-F type stars ${ }^{\star, \star \star, \star \star \star}$
}

\section{A planetary system found with HARPS around the F6IV-V star HD 60532}

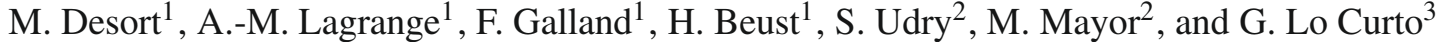 \\ ${ }^{1}$ Laboratoire d'Astrophysique de Grenoble, UMR 5571 CNRS, Université Joseph Fourier, BP 53, 38041 Grenoble Cedex 9, France \\ e-mail: morgan.desort@obs.ujf-grenoble.fr \\ 2 Observatoire de Genève, Université de Genève, 51 chemin des Maillettes, 1290 Sauverny, Switzerland \\ 3 European Southern Observatory, Alonso de Cordova 3107, Vitacura, Santiago, Chile
}

Received 22 May 2008 / Accepted 22 September 2008

\section{ABSTRACT}

\begin{abstract}
Aims. In the framework of the search for extrasolar planets and brown dwarfs around early-type stars, we present the results obtained for the F-type main-sequence star HD 60532 (F6V) with HARPS.

Methods. Using 147 spectra obtained with HARPS at La Silla on a time baseline of two years, we studied the radial velocities of this star.

Results. HD 60532 radial velocities are periodically variable, and the variations have a Keplerian origin. This star is surrounded by a planetary system of two planets with minimum masses of 1 and $2.5 M_{\text {Jup }}$ and orbital separations of 0.76 and 1.58 AU, respectively. We also detect high-frequency, small-amplitude $\left(10 \mathrm{~m} \mathrm{~s}^{-1}\right.$ peak-to-peak) pulsations. Dynamical studies of the system point toward a possible 3:1 mean-motion resonance that should be confirmed within the next decade.
\end{abstract}

Key words. techniques: radial velocities - stars: early-type - stars: planetary systems - stars: individual: HD 60532 stars: oscillations

\section{Introduction}

Radial-velocity (RV) surveys have lead to the detection of nearly 300 planets $^{1}$ during the past decade. They mainly focus on solar and late-type main-sequence (MS) stars ( $\gtrsim F 7)$ that exhibit numerous lines with small rotational broadening. It is often thought that planets around more massive MS stars are not accessible to radial-velocity techniques, as they present a small number of stellar lines, usually broadened and blended by stellar rotation. However, we recently showed (Galland et al. 2005a, Paper I) that with a new radial-velocity measurement method that we developed, it is possible to detect planets even around early A-type MS stars with high rotational velocities (typically $100 \mathrm{~km} \mathrm{~s}^{-1}$ ). Finding planets around such massive MS stars is important, as this allows planetary formation and evolution processes to be tested around a wide variety of stars, in terms of stellar mass and time scales of evolution processes. This approach is complementary with the one which intends to detect planets around evolved intermediate-mass stars (e.g., Sato et al. 2005;

\footnotetext{
* Appendix A is only available in electronic form at http://www . aanda.org

$\star \star$ Based on observations collected at the European Organisation for Astronomical Research in the Southern Hemisphere, Chile, ESO. Program IDs 072.C-0488, 076.C-0279, 077.C-0295, 078.C-0209, 080.C-0664, 080.C-0712, 081.C-0774.

$\star \star \star$ Table of radial velocities is only available in electronic form at the CDS via anonymous ftp to

cdsarc.u-strasbg.fr (130.79.128.5) or via

http://cdsweb.u-strasbg.fr/cgi-bin/qcat?J/A+A/491/883

1 Jean Schneider, http://exoplanet.eu
}

Lovis \& Mayor 2007). In this case, close-in planets have been wiped out but the stellar variability is in principle less intense.

We performed a radial-velocity survey dedicated to the search for extrasolar planets and brown dwarfs around a volume-limited sample of $\mathrm{A}-\mathrm{F}$ main-sequence stars with the HARPS spectrograph (Mayor et al. 2003) installed on the 3.6-m ESO telescope at La Silla Observatory (Chile). We monitored a sample of $185 \mathrm{MS}$ stars with $B-V$ ranging between -0.1 and 0.6. From the measured RV jitter, we computed the minimum detectable masses with HARPS, and showed that in 100 cases, planets with periods shorter than 100 days can be detected, even around stars with early spectral types (down to $\sim 0.1 M_{\text {Jup }}$ at 100 days around slow-rotating late-F stars). Given the data at hand, we also provided the achieved detection limits on the individual targets (Lagrange et al. 2008).

In the course of this survey, we identified a few stars whose RV variations could be attributed to planets. Most of these stars are still being followed up on. We present here the detection of a planetary system around one of these stars, HD 60532. Section 2 provides the stellar properties of this star, the measurement of the radial velocities, and their relevance. We also present a Keplerian solution associated to the presence of two planets. In Sect. 3 we discuss the dynamical stability of the system.

\section{Stellar characteristics and measurements}

\subsection{Stellar properties}

HD 60532 (HIP 36795, HR 2906) is located at $25.7 \mathrm{pc}$ from the Sun (ESA 1997). Stellar parameters such as mass, age, metallicity, rotational velocity, and effective temperature are taken from 
Table 1. HD 60532 stellar properties ${ }^{a}$.

\begin{tabular}{llc}
\hline \hline Parameter & & HD 60532 \\
\hline Spectral type & & F6IV-V \\
$v \sin i$ & {$\left[\mathrm{~km} \mathrm{~s}^{-1}\right]$} & 8 \\
$V$ & & 4.45 \\
$B-V$ & & 0.52 \\
$\pi$ & {$[\mathrm{mas}]$} & $38.9 \pm 0.7$ \\
Distance & {$[\mathrm{pc}]$} & 25.7 \\
$M_{V}$ & & 2.40 \\
$\log R_{\mathrm{HK}}^{\prime}$ & & -4.94 \\
{$[\mathrm{Fe} / \mathrm{H}]$} & & -0.42 \\
$T_{\text {eff }}$ & {$[\mathrm{K}]$} & 6095 \\
$\log g$ & {$\left[\mathrm{~cm} \mathrm{~s}{ }^{-2}\right]$} & -3.83 \\
$M_{1}$ & {$\left[M_{\odot}\right]$} & $1.44_{-0.1}^{+0.03}$ \\
Age & {$[\mathrm{Gyr}]$} & $2.7 \pm 0.1$ \\
\hline
\end{tabular}

${ }^{a}$ Photometric and astrometric data are extracted from the HIPPARCOS catalogue (ESA 1997); spectroscopic data are from Nordström et al. (2004).

Nordström et al. (2004), and the gravity is taken from Gray et al. (2006). Those values are reported in Table 1. They agree with a spectral type F6IV-V (F6IV in the Bright Star Catalogue, Hoffleit et al. (1991), F6V in the HIPPARCOS catalogue (ESA 1997).

\subsection{Radial-velocity measurements}

Since February 2006, 147 high signal-to-noise ratio $(S / N)$ spectra have been acquired with HARPS, with a $S / N$ equal to 310 on average. Each spectrum is formed by 72 spectral orders covering the spectral window ( $3800 \AA$, $6900 \AA$ ), with a resolution $R \approx 115000$.

The radial velocities and associated uncertainties have been measured with a dedicated tool (SAFIR) which uses the Fourier interspectrum method described in Chelli (2000) and in Galland et al. (2005a). The uncertainty is equal to $0.9 \mathrm{~m} \mathrm{~s}^{-1}$ on average, consistent with the value obtained from our simulations (see Paper I), and includes photon noise, instrumental effects and guiding errors (fixed at a upper limit of $0.5 \mathrm{~m} \mathrm{~s}^{-1}$ ).

Note: given the star spectral type, we could also derive the RV from Gaussian adjustements to the CCFs (Cross-Correlation Functions), using a mask with a G2 spectral type. The obtained values are compatible within the error bars with the ones measured by SAFIR.

The amplitude of the radial-velocity variations (more than $\left.120 \mathrm{~m} \mathrm{~s}^{-1}\right)$ as well as their standard deviation $\left(\sigma_{\mathrm{RV}}=\right.$ $27 \mathrm{~m} \mathrm{~s}^{-1}$ ) are much larger than the uncertainties. We now show that these variations are not due to line-profile variations and we interpret them in terms of the presence of planetary companions.

The periodogram (Fig. 1) of the RVs indicates four main periodicities in the data. Two of them (near 30 days) are aliases of data (Fig. 1, bottom represents the alias generated by our sampling frequency), whereas the other two ( 220 and 500-1000 days) are real periodicities of the RVs. This points toward the existence of two companions in orbit with periods close to those one.

\subsection{A Keplerian origin to the main variations}

In a (RV; bisector velocity span) diagram (Fig. 2), the bisector velocity spans are spread mainly horizontally and are not correlated with the RVs. This argues in favour of a planetary origin to the variations rather than stellar activity (spots, pulsations).

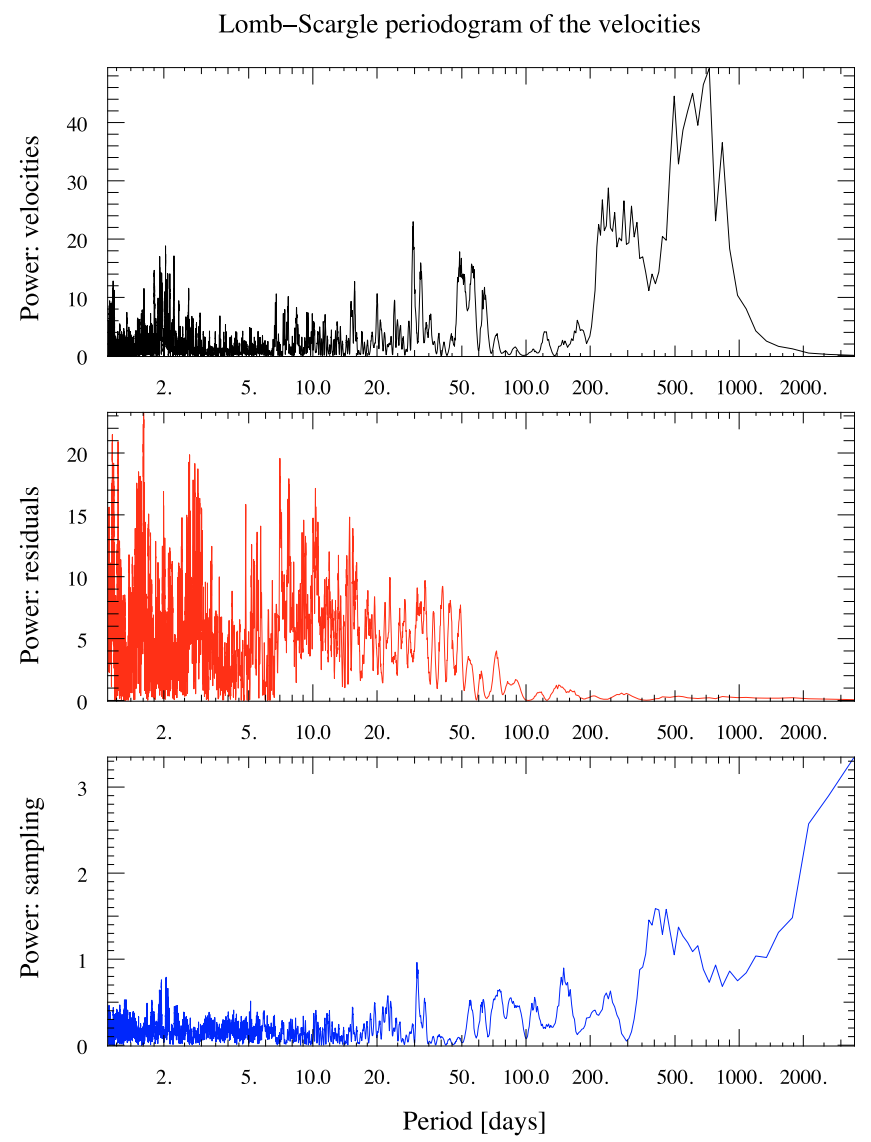

Fig. 1. Periodogram of the RVs (top). Four main peaks are visible. The two near 30 days are observation alias (bottom, periodogram of the observation epochs), the other two correspond roughly to periods of $\sim 220$ and $\sim 500-1000$ days. These periods are not well constrained because of the limited phase coverage (our data span only over $\sim 900$ days. The middle panel shows the periodogram of the residuals to the final keplerian solution.

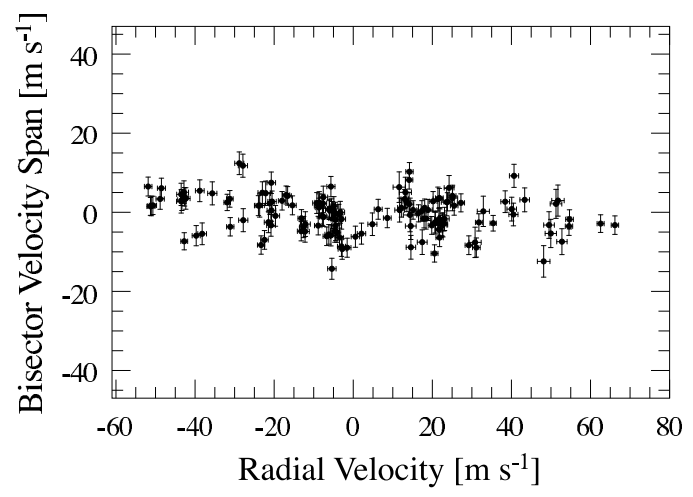

Fig. 2. Bisector velocity spans versus RVs. The bisector velocity spans lie within $\pm 15 \mathrm{~m} \mathrm{~s}^{-1}$ whereas the RVs vary over more than $120 \mathrm{~m} \mathrm{~s}^{-1}$ without correlation (see Sect. 2.3).

A spot origin can be rejected from several grounds: 1) given the $\operatorname{star} v \sin i$, much higher than the instrumental resolution $\left(8 \mathrm{~km} \mathrm{~s}^{-1}\right.$ compared to $3 \mathrm{~km} \mathrm{~s}^{-1}$ ), cool spots on the surface of the star would induce a correlation between bisector velocity spans and radial velocities (Desort et al. 2007), and the RV variations would have a period similar to the one of the star's rotational pe$\operatorname{riod}^{2}$, whereas they are actually much longer; 2) also, spots able

\footnotetext{
${ }^{2}$ Here, the rotational period of the star $P_{\text {rot }}$ is lower than $\sim 7$ days.
} 


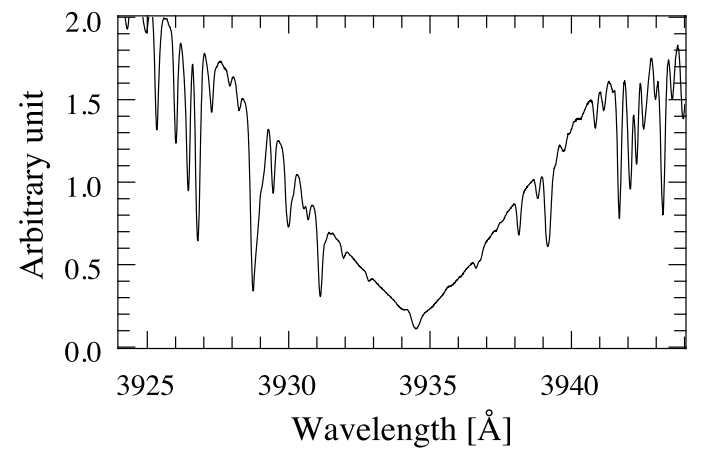

Fig. 3. No emission in the Ca II K line for the HD 60532 spectra. This is the average spectrum of all the spectra used after recentring by the $\mathrm{RV}$ variations.
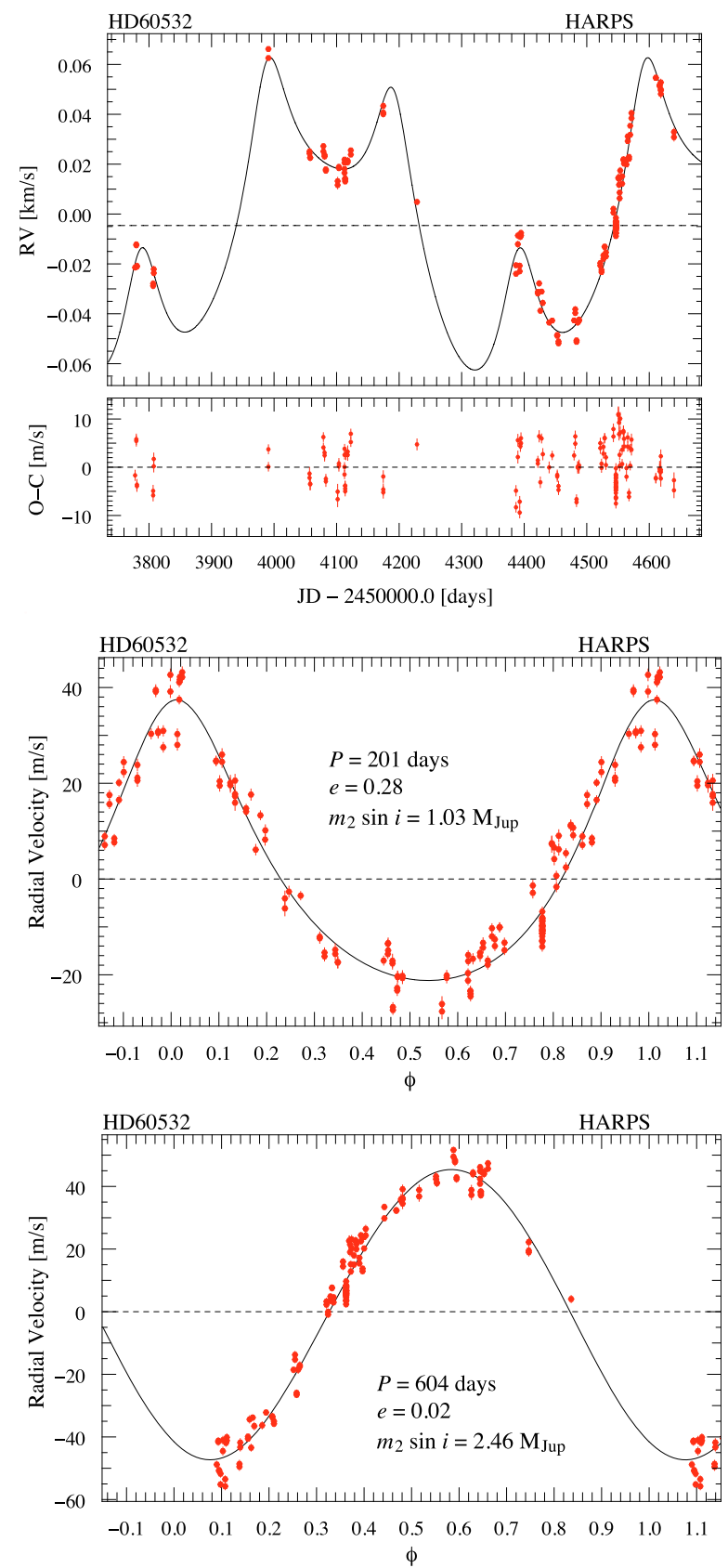

Fig. 4. Top: HARPS radial velocities and orbital solution for HD 60532 , the panel below shows the residuals to the fitted orbital solution. Middle and bottom: phased fits on individual planets.
Table 2. Best orbital solution for HD 60532.

\begin{tabular}{llcc}
\hline \hline Parameter & & Planet $b$ & Planet $c$ \\
\hline$P$ & [days] & $201.3 \pm 0.6$ & $604 \pm 9$ \\
$T_{0}$ & {$[\mathrm{JD}-2450000]$} & $3987 \pm 2$ & $3723 \pm 158$ \\
$e$ & & $0.28 \pm 0.03$ & $0.02 \pm 0.02$ \\
$\omega$ & {$[\mathrm{deg}]$} & $-8.1 \pm 4.9$ & $-209 \pm 92$ \\
$K$ & {$\left[\mathrm{~m} \mathrm{~s}^{-1}\right]$} & $29.3 \pm 1.4$ & $46.4 \pm 1.7$ \\
$N_{\text {meas }}$ & & 147 & - \\
$\sigma_{O-C}$ & {$\left[\mathrm{~m} \mathrm{~s}^{-1}\right]$} & 4.4 & - \\
reduced $\chi^{2}$ & & 4.4 & - \\
\hline$a_{1} \sin i$ & {$\left[10^{-3} \mathrm{AU}\right]$} & 0.52 & 2.6 \\
$f(m)$ & {$\left[10^{-9} M_{\odot}\right]$} & 0.46 & 6.2 \\
$M_{1}$ & {$\left[M_{\odot}\right]$} & 1.44 & - \\
$m_{2} \sin i$ & {$\left[M_{\text {Jup }}\right]$} & $1.03 \pm 0.05$ & $2.46 \pm 0.09$ \\
$a$ & {$[\mathrm{AU}]$} & $0.759 \pm 0.001$ & $1.58 \pm 0.02$ \\
\hline
\end{tabular}

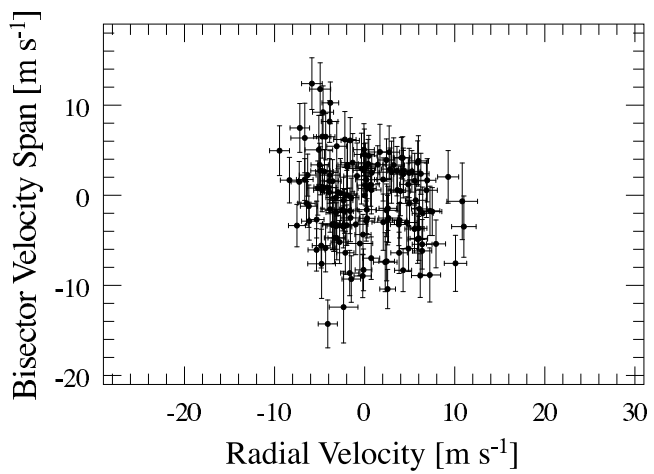

Fig. 5. (O-C; O-C bisector velocity span) diagram: the $\mathrm{O}-\mathrm{C}$ bisector velocity spans are not horizontally spread (Sect. 2.5).

to produce such amplitude of RV variations would induce detectable photometric variations, whereas the photometry given by HIPPARCOS (ESA 1997) is constant with a scatter of only $0.004 \mathrm{mag} ; 3$ ) the star has a low level of activity (no emission in the Ca II K line, Fig. 3, and $\left.\operatorname{low} \log R_{\mathrm{HK}}^{\prime}\right)^{3}$.

\subsection{Orbital parameters}

The observed RV can clearly not be fitted by one companion. The orbital parameters derived from the best two-companion Keplerian solution (Fig. 4) are given in Table 2. The residuals dispersion is $4 \mathrm{~m} \mathrm{~s}^{-1}$. A planetary system of two Jupiter-mass planets on long-period orbits fits the data very well. Assuming a primary mass of $1.44 M_{\odot}$, this leads to a system with a $2.5-M_{\text {Jup }}$ planet on a $604-$ day orbit and a $1.0-M_{\text {Jup }}$ planet on a 201-day orbit.

\subsection{Interpretation of the residuals}

The residuals to the possible orbital solutions show variations, with standard deviation of $4.4 \mathrm{~m} \mathrm{~s}^{-1}$; we do not find any periodicity in these variations. The amplitude of variations is the same as the level of the small-amplitude variations seen in the line profile. Also, in a (O-C; O-C bisector velocity span) diagram, the $\mathrm{O}-\mathrm{C}$ bisector velocity spans are not horizontally spread, which shows that these remaining variations are not due to the presence of a smaller-mass planet (Fig. 5). They are then probably due to stellar intrinsic phenomena. We monitored the star continuously

\footnotetext{
${ }^{3}$ See online section for a figure showing the bisectors of all the spectra and for an example of an active, not young F6V star.
} 

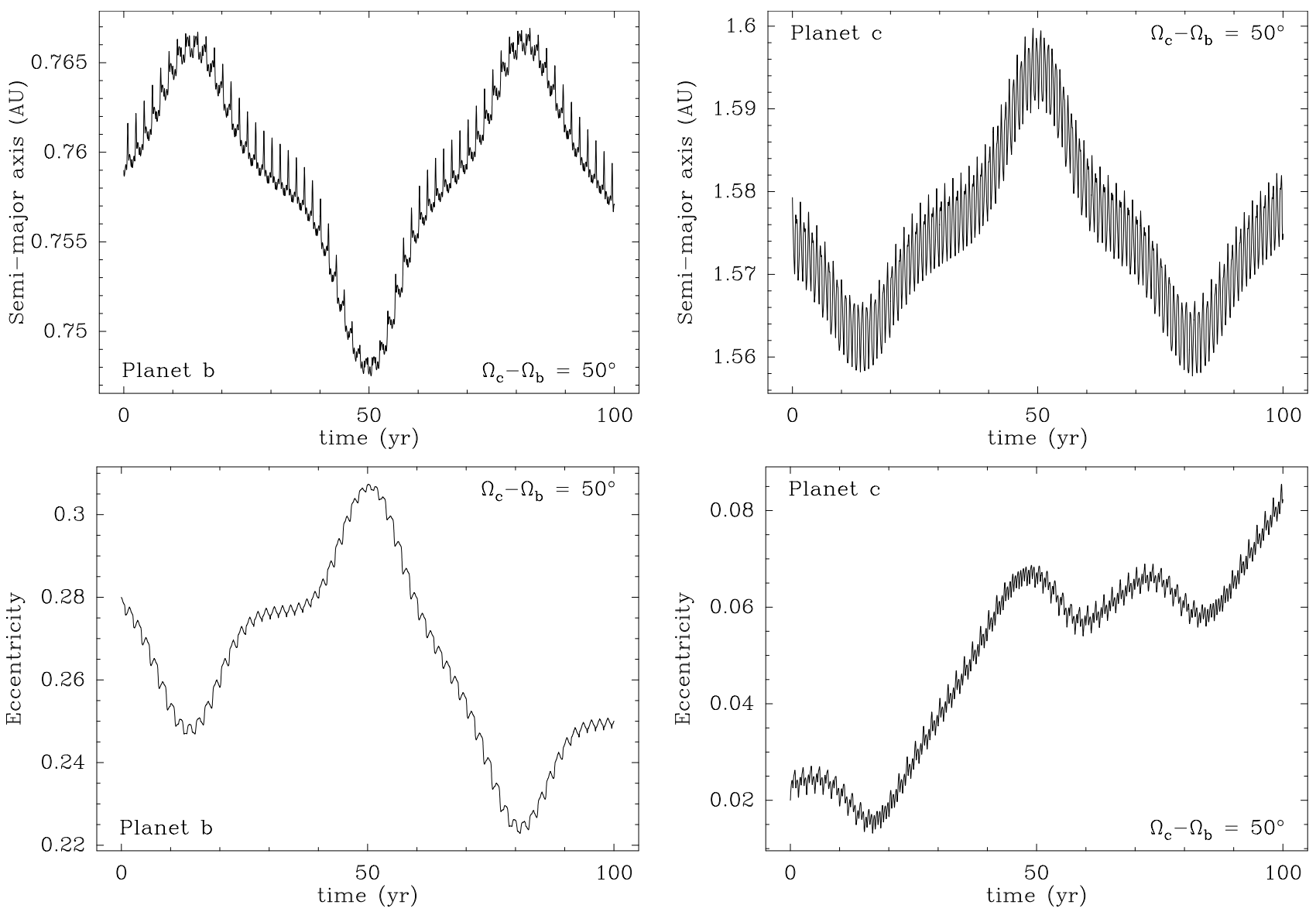

Fig. 6. Orbital evolution over $100 \mathrm{yr}$ of the semi-major axes (top) and eccentricities (bottom) for planets $b$ (left) and $c$ (right), under their mutual perturbations, in a 3:1 resonance configuration, for an initial choice of $\Omega_{c}-\Omega_{b}=50^{\circ}$.

for one hour (28 spectra) and measured RV variations with a peak-to-peak amplitude of $9 \mathrm{~m} \mathrm{~s}^{-1}$. This corresponds to the observed semi-amplitude of the residuals. Such high-frequency variations argue for stellar pulsations and if we would like to increase the measurement accuracy we would need to average this effect. Finally, we could not find any periodicity associated with the residuals.

\section{Dynamical issues}

The two orbital periods derived from the fit suggest a possible 3:1 mean-motion resonance between the two planets. Here we investigate the dynamical behaviour of the system to possibly distinguish between resonant and non-resonant configurations. As we show below, the uncertainty on the semi-major axis values for planets $b$ and $c$ (mainly on Planet $c$, see Table 2) does not permit us to state whether the planets are actually locked in mean-motion resonance of just next to it.

The two planets are said to be in mean-motion resonance when their orbital periods achieve a simple rational ratio. More generally, they will be assumed to be locked in a $p+q: p$, where $p$ and $q$ are integers. $|q|$ is called the order of the resonance, and denotes the number of stable conjunction positions. In the 3:1 case, we have $q=2$. The dynamics within a resonance is usally described via the use of the following variable called critical argument of the resonance:

$\sigma=\frac{p+q}{q} \lambda_{c}-\frac{p}{q} \lambda_{b}-\varpi_{b}$ where $\lambda_{b}$ and $\lambda_{c}$ are the mean longitudes of the inner and the outer planet, respectively, and where $\varpi_{b}$ is the longitude of periastron of the inner planet (here the less massive one). Resonant orbits are characterised by a libration of $\sigma$ around an equilibrium position $\sigma_{0}$, while non-resonant orbit exhibit a circulation of $\sigma$. In non-resonant configurations, the semi-major axes are nearly secular invariants, due to independent phase averaging over both orbits, while in resonant configurations, the $\sigma$ libration induces secular eccentricity and semi-major axis oscillations.

We integrate the 3-body system (star +2 planets, assuming $\sin i=1$ ) starting from the today fitted orbital solution, using the symplectic $N$-body code SyMBA (Duncan et al. 1998). The use of a powerful symplectic code allows us to adopt a large timestep while remaining accurate. We adopt here a timestep of $0.025 \mathrm{yr}$, i.e., a bit less than $1 / 20$ of the lowest orbital period. This is a standard prescription in the use of this code to ensure a relevant accuracy. We also made tests using a ten times lower timestep to check the validity of our integrations. From a dynamical point of view the orbital solution as given in Table 2 is not complete. The longitude of nodes $\Omega_{b}$ and $\Omega_{c}$ are not constrained. Note that thanks to rotational invariance, only the difference $\Omega_{c}-\Omega_{b}$ is a relevant parameter. It will be treated as a free parameter in our integrations.

We first focus on resonant configurations. For this we assume for the semi-major axes $a_{b}=0.759 \mathrm{AU}$ and $a_{c}=1.57928 \mathrm{AU}$. This choice for $a_{c}$ (within the error bar) ensures an exact resonant configuration.

Figure 6 shows the orbital evolution over $100 \mathrm{yr}$ of the two planets in this configuration under their mutual perturbations, for 


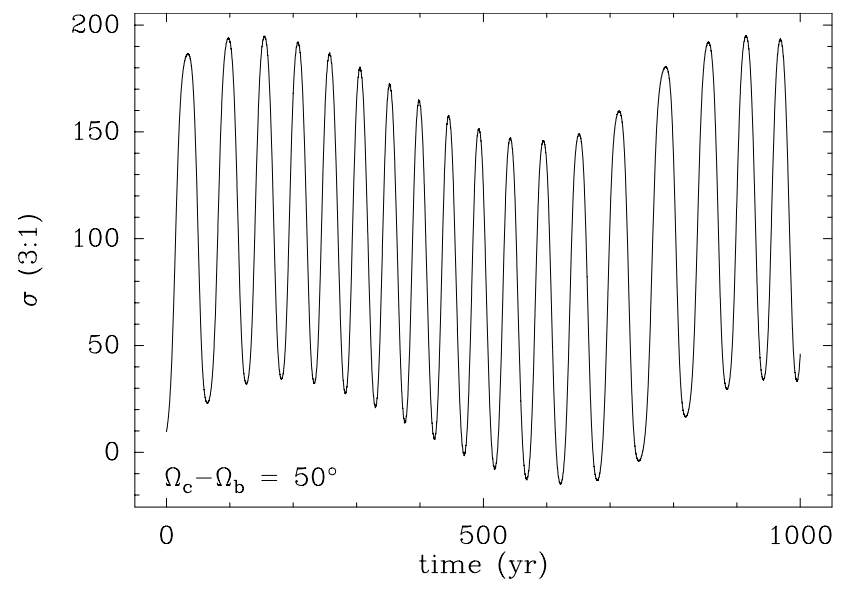

Fig. 7. Evolution of the $3: 1$ critical argument $\sigma$ over $1000 \mathrm{yr}$, in the same condition as described in Fig. 6. We note the $\sigma$-libration characteristic for resonant motion.
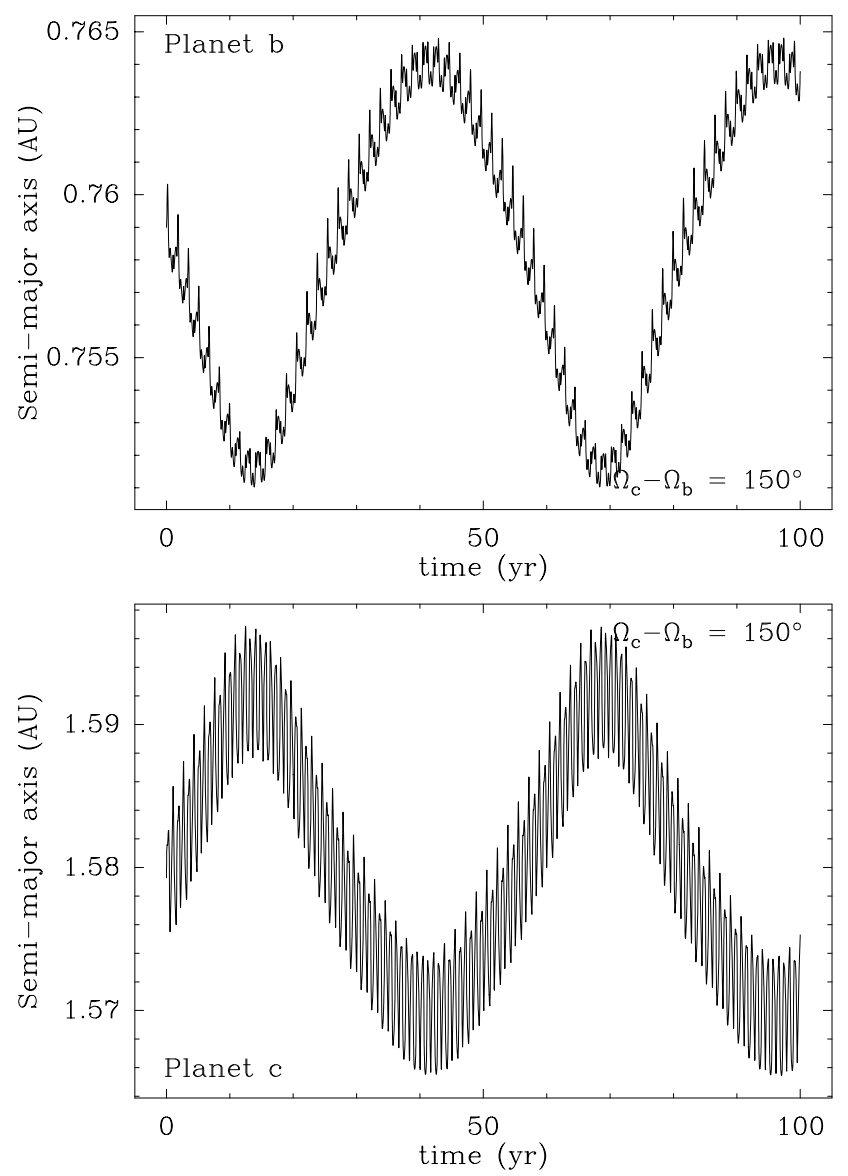

Fig. 8. Evolution of the semi-major axes of the two planets in the same condition as described in Fig. 6, except that we assumed $\Omega_{c}-\Omega_{b}=150^{\circ}$.

a choice of $\Omega_{c}-\Omega_{b}=50^{\circ}$. We note that both the semi-major axes and the eccentricities exhibit a superimposition of two types of variations: we first have a long-period oscillation with $\sim 70 \mathrm{yr}$ period, in addition to high frequency (1-2 yr periodicity), but much smaller amplitude changes. The former oscillation is related to the resonant libration motion (see Fig. 7), while the latter is phased with the synodic motion of the two planets. It is thus related to the mutual perturbations of the two bodies at conjunction. The fact that this high-frequency term shows up here is an indication for weak - but significant - chaos in the system.
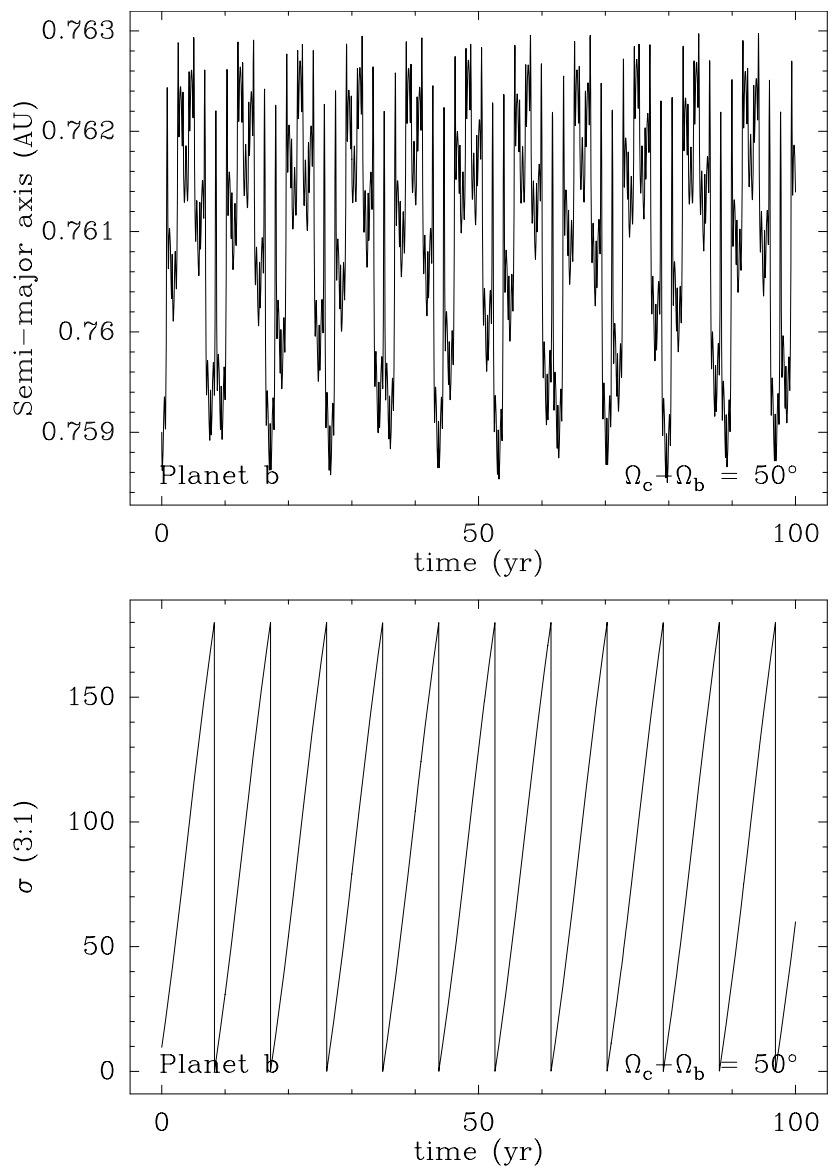

Fig. 9. Evolution of the semi-major axis of Planet $b$ and of the critical angle $\sigma$ for 3:1 resonance in the same conditions as described in Fig. 6, but with $a_{c}=1.57528 \mathrm{AU}$. This is a non-resonant configuration.

The integration was extended up to $10^{8} \mathrm{yr}$. Over this time span, the planetary system does not show any indication for instability. The semi-major axes of the two planets oscillate between 0.748 AU and 0.767 AU for Planet $b$, and between 1.558 AU and 1.6 AU for Planet $c$. The eccentricity of Planet $b$ oscillates between 0.13 and 0.32 , and that of Planet $c$ between 0 and 0.14 . Note that the present value of Planet $c$ 's eccentricity is close to the bottom of its actual variation range.

Figure 6 shows that, given the error bars listed in Table 2, the secular evolution of the semi-major axis of Planet $b$ should be detectable within $\sim 10 \mathrm{yr}$ from now. In fact, what is expected to be detected is only the low-frequency secular variation. The high-frequency oscillation remains below the error bar.

Now, we point out that Fig. 6 corresponds to $\Omega_{c}-\Omega_{b}=50^{\circ}$. Figure 8 shows the evolution of the semi-major axes in the same conditions, but starting with $\Omega_{c}-\Omega_{b}=150^{\circ}$. We still see the same kind of oscillations, but phased differently. In Fig. 6, the semi-major axis of Planet $b$ increases in the first 10 years, while in Fig. 8, it decreases.

Although the actual value of $\Omega_{c}-\Omega_{b}$ is unknown, the detection of semi-major axis evolution of Planet $b$ within 10 years should be considered as a strong indication of a resonant configuration. Figure 9 shows the evolution of the semi-major axis of Planet $b$ and of the critical angle $\sigma$ for 3:1 resonance for an initial choice of $a_{c}=1.57528 \mathrm{AU}$. As can be seen from the evolution (circulation) of $\sigma$, this configuration is non-resonant, while still within the error bar of the orbital fit. Hence, from the orbital fit itself, it is not possible to definitely state whether the two planets 
are actually locked in 3:1 resonance or not. From Fig. 9, we see that in non-resonant configuration, the variations of the semimajor axis of Planet $b$ achieve a much smaller amplitude than in the resonant case. This remains true for any choice of initial non-resonant configuration. In fact, in that case, we only have the high-frequency, small-amplitude oscillation. As expected in non-resonant case, there is no secular evolution of the semimajor axis, contrary to the resonant case. The net consequence of this is that, given the error bars of the fit, no semi-major axis variation for Planet $b$ should be detected within 10 years in the non-resonant case.

Our conclusions concerning this dynamical study are thus the following:

- given the error bars of the fit, it is not possible to definitely state the planets are actually locked in mean-motion resonant or not;

- the two-planet system is dynamically stable although significantly chaotic;

- although it is impossible to predict the variation sense due to unconstrained $\Omega_{c}-\Omega_{b}$, any detection of variation in the semimajor axis of Planet $b$ within 10 years from now should be a strong indication for a resonant configuration. Further monitoring of this system should therefore be initiated to detect this possible variation.

\section{Concluding remarks}

We have shown that HD 60532, an F6IV-V, $1.44 M_{\odot}$ star hosts two planets with minimum masses of 1 and $2.5 M_{\text {Jup }}$ and orbital separations of 0.76 and $1.58 \mathrm{AU}$ respectively, in a possible
3:1 resonance which needs to be confirmed within the next 10 years. Noticeably sofar, only one other multiple system had been reported around a MS star more massive than $1.3 M_{\odot}\left(\mathrm{HD} 169830 ; 1.4 M_{\odot}\right)$. Note also that the low metallicity of HD 60532 is not common for stars harbouring Jupiter-mass planets; the relation between the star's metallicity and the presence of massive planets has still to be investigated further.

Acknowledgements. We acknowledge financial support from the French Agence Nationale pour la Recherche, ANR grant NT05-4 44463. We are grateful to ESO and the La Silla Observatory for the time allocation and to their technical staff. We also thank the HARPS GTO observers for performing some observations, Gérard Zins and Sylvain Cètre for their help in implementing the SAFIR interface. These results have made use of the SIMBAD database, operated at CDS, Strasbourg, France.

\section{References}

Chelli, A. 2000, A\&A, 358, L59

Desort, M., Lagrange, A.-M., Galland, F., et al. 2007, A\&A, 473, 983

Duncan, M. J., Levison, H. F., \& Lee, M. H. 1998, AJ, 116, 2067

Galland, F., Lagrange, A.-M., Udry, S., et al. 2005a, A\&A, 443, 337

Gray, R. O., Corbally, C. J., Garrison, R. F., et al. 2006, AJ, 132, 161

ESA 1997, The Hipparcos and Tycho Cat, ESA SP-1200

Hoffleit, D., \& Warren Jr., W. H. 1991, Bright Star Catalogue (5th Revised edn.), NSSDC/ADC

Lagrange, A.-M., Desort, M., Galland, F., et al. 2008, A\&A, accepted [arXiv: 0809.4636]

Lovis, C., \& Mayor, M. 2007, A\&A, 472, 657

Mayor, M., Pepe, F., Queloz, D., et al. 2003, The Messenger, 114, 20

Nordström, B., Mayor, M., Andersen, J., et al. 2004, A\&A, 418, 989

Sato, B., Kambe, E., Takeda, Y., et al. 2005, PASJ, 57, 97 
M. Desort et al.: Extrasolar planets and brown dwarfs around A-F type stars, Online Material p 1

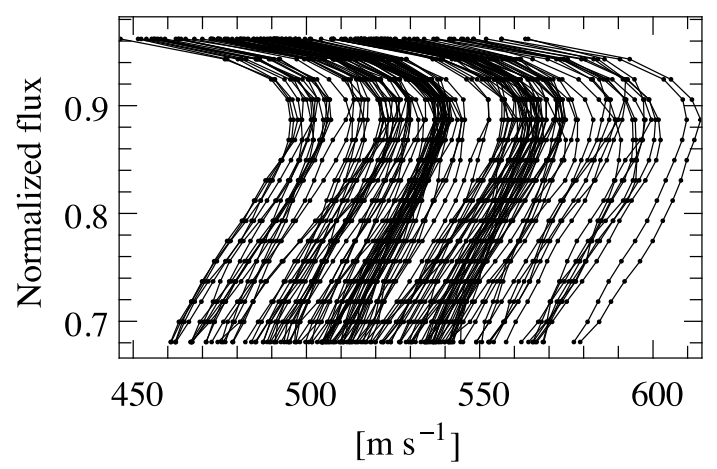

Fig. A.1. Bisectors of the CCFs.
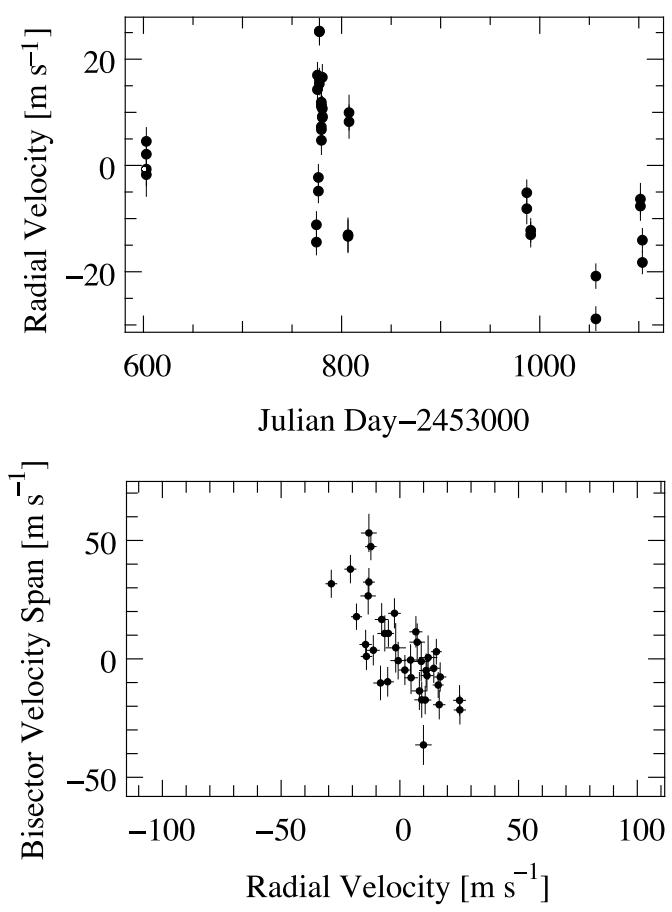

Fig. A.2. Example of an active F6V star for which measured RVs (top) are correlated to the bisector velocity spans (bottom).

\section{Appendix A: Additional material}

Figure A.1 presents the bisectors of the CCFs (which stands for all the lines) for the spectra from which we measured the radial velocities of HD 60532. It shows that spectra are mainly shifted, with yet small line-profile variations superimposed to the shifts, and thus that the radial velocities measured are not induced by line-profile variations.

Figure A.2 shows an example of an active F6V star (HD 30652, $v \sin i=16 \mathrm{~km} \mathrm{~s}^{-1}$ ) that we observed during the same survey, and for which RV measurements are correlated with lineprofile variations, indicating that they are are induced by a spot. 